\title{
Restoring and Enhancing Degraded Underwater Pipelines for Identifying and Detecting Corrosion
}

\author{
Vaibhav A. Parjane ${ }^{\mathrm{a}, 1}$ and Dr. Mohit Gangwar ${ }^{\mathrm{b}}$ \\ ${ }^{a}$ Ph.D. Research Scholar, Department of Computer Science and Engineering, Bhabha University, Bhopal, \\ Madhya Pradesh, India \\ ${ }^{b}$ Dean-Engineering, Bhabha University, Bhopal, Madhya Pradesh, India
}

\begin{abstract}
Detection of corrosion from underwater images is necessary for oil and gas pipelines to eliminate the internal leakages and hazards. The tests utilized a broad range of underwater pictures of various situations. A modern technique for estimating subsea pipeline corrosion based on the colour of the corroded pipe. For corrupted underwater videos, an image reconstruction and enhancement algorithm is created as a preliminary phase. The created algorithm reduces blurring and improves picture colour and contrast. The improved colours in the imaging details aid in the method of corrosion estimation. In this work we proposed a underwater corrosion detection using image processing techniques. Some machine learning and deep learning techniques have been used for classification of corrosion. In experimental analysis various features have been evaluated for detection of corrosion and it introduces better classification accuracy than traditional approaches.
\end{abstract}

Keyword. Corrosion detection, Image process, Deep Learning, DCNN

\section{Introduction}

Therefore, we need detection and testing techniques based on deep learning methods that alert us to the first access to water in surface images and insulation. Deep learning to prepare rust estimates and develop perspectives. Create different corrosion picture parameters of the pipeline under different tests and estimate which utility does not count the execution of trains and testing with different fold assumptions. Exterior sunshine is caused by internal pipeline oxidation, resulting in general corrosion Due to thin internal walls (homogeneous rust), pitting (local rust), and fracture rust, and microbiological rust. The most common problem is insulation repair (ICC) in the oil and gas industry. In the proposed research work, using a deep learning algorithm, design and implement a system for detecting and predicting corrosion on the data set of the underwater pipeline image. The system uses different deep learning algorithms to classify algorithms and predict the possibility of detecting corrosion.

\footnotetext{
${ }^{1}$ Vaibhav A. Parjane, Ph.D. Research Scholar, Department of Computer Science and Engineering, Bhabha University

Email.parjanevaibhav77@gmail.com.
} 


\section{Literature Survey}

There are many techniques that are being used today to analyze CUI. None of the techniques was applied on their own. For the best performance, several strategies should be used in conjunction with one another. Corrosion under insulation can be detected in many ways, though most is by checking the placement of the connection between the cable and the device. In order to enable ultrasonic testing, it must be expunged. Radiography and total separation are two others widely used approaches. These approaches require the use of a Pulse Eddy Current. The following are some of the most popular techniques for examining CUIs.

\subsection{Existing Methodologies}

Red-dark channels were previously defined and removed for background light and estimation of the outbreak of this disease. Visualization compensation for objectcamera distance retrieval Background and color of objects by analyzing the physical properties of We developed a simple but efficient low-pass filter to debug the point spread function, Debiler Underwater imagery. Different types of water surface images were used under different conditions for experiments. The experimental results indicate that the proposed algorithm effectively Underwater images were recovered while absorbing and dispersing effects [1-4].

The reason for damaging the surface image of the water is to survey sophisticated intelligence algorithms. As a sophisticated method of deicing and refining underwater images, underwater image decorating performance, and color restoration in various ways, the underwater image identifies color evaluation metrics and provides an overview of the critical underwater image applications. Underwater environment, which contains numerous organisms Resources and energy are the main factors needed to sustain sustainability Human development. People often use videos or images to get valuable information when studying the underwater environment. Underwater imagery is the enhancement of contrast widely used techniques for color correction. Contrast is an enhancement of contrast that has attracted much attention in recent years [5-9].

Exploitation is advantageous because they have the ability to use side-scan sonar (SS) for testing underwater meteoric like, i.e., explode (cables and pipelines). When designing houseboats, builders propose (supposedly) to use water vessels that have their own purposes and goals. The issue is fixing the contact lines and under water depth barriers when trying to identify the fixed points of the robots [10]. The original study details of the cable quest results and of the Pipeline simulation experiments are reviewed. An android body that can be programed the work area is small because they have to account for the effect of other elements, and because additional characters are required (supporting characters are required). The effect of realising thematic seabed scenes using AUV Cable measurements (measurements obtained by the AUV) According to the testing of this device, we know that this can be used in an acoustic control system in underwater robots, the use of the pipeline monitoring system in the investigation of movement is supported pressed for change. 


\section{Proposed System Details}

\subsection{Problem Statement}

In the proposed research work, using deep learning algorithm, design and implement a system for detecting and predicting corrosion on the data set of the underwater pipeline image. The system uses different deep learning algorithms to classify algorithms as well as to predict the possibility of detecting corrosion.

\subsection{System Functions}

- Creating and developing approaches for corrosion prediction models for oil and gas pipelines using underwater imagery and deep learning approaches.

- Design and build rust detection systems using water-influencing pipeline images.

- Developing an algorithm to detect harmful patches from input images using the DCNN algorithm deep learning foundation.

- Defining the image restoration technique using the CNN approach.

- Exploring and validating the proposed efficacy of the program with certain existing systems.

\subsection{System Architecture}

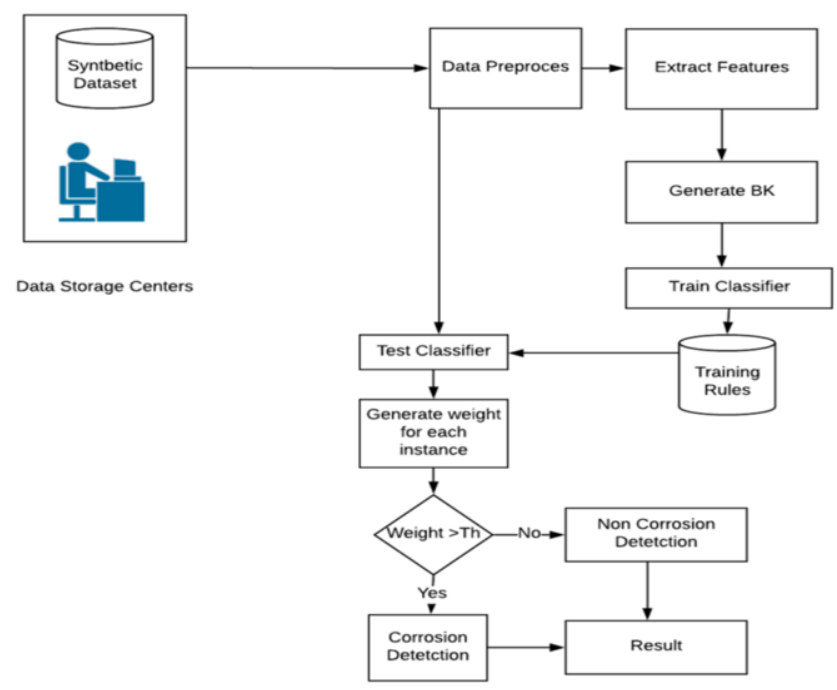

Figure 1. Proposed system architecture

The BK is nothing but a background knowledge that is generated based on extracted values from sensing systems. According to the proposed algorithm, each event gives reward or penalty respectively, based on that each event changes weight state, and based on that system generates BK rules during the execution. 


\subsection{Algorithm Design}

Input. Test Dataset which contains various test instances Test DBLits [], Train dataset which is built by training phase Train_DBLits[], Threshold Th.

Step 1. For each read each test instances using below equation TestFeature $(\mathrm{m})=$ $\sum_{m=1}^{n}($ featureSet $[\mathrm{A}[\mathrm{i}] \ldots \ldots \ldots \mathrm{A}[\mathrm{n}]$ TestDBList)

Step 2. Extract each feature as a hot vector or input neuron from test-Feature $(\mathrm{m})$ using below equation.

Extracted_Feature $[\mathrm{t} . . . . . . \mathrm{n}]=\sum_{m=1}^{n}((\mathrm{t}) \leftarrow$ testFeature $)$

Extracted-Feature-Set $x[t]$ contains the feature vector of respective domain

Step3. For each read each train instances using below equation

Train_Feature $(\mathrm{m})=\sum_{m=1}^{n}($ featureSet $[\mathrm{A}[\mathrm{i}] \ldots \ldots \ldots \mathrm{A}[\mathrm{n}]$ TestDBList $)$

Step 4. Extract each feature as a hot vector or input neuron from test-Feature $(\mathrm{m})$ using below equation.

Extracted_Feature [t........n] $=\sum_{m=1}^{n}((\mathrm{t}) \leftarrow$ trainFeature $)$

Extracted-Feature-Set $\mathrm{x}[\mathrm{t}]$ contains the feature vector of respective domain.

Step 5. Now map each test feature set to all respective training feature set

Weight $=$ Calscore $\left(\right.$ FeatureSet $X \| \sum_{m=1}^{n}($ featureSety $[\mathrm{y}])$

Step 6. return instance [label [weight]

Output. HashMap <class label, Similarity_Weight $>$ all instances which weight violates the threshold score.

\section{Results and Discussions}

For experiment analysis of proposed system evaluate entire execution in two different open-source platforms. First system creates network simulator environment to generate sensor nodes, the entire simulation log has used as IoT communication log which is generated by various analogue sensors. The techniques basically define in base approach which is carried out to calculate the various parameters between two sensors called train and test feature vectors weight. For each transaction system automatically calculate some values which is denoted in matrix X. K-means clustering unsupervised learning approach has used to generate the data labels and DCNN has used as a supervised learning algorithm.

Table 1. Performance evaluation with DCNN and SVM

\begin{tabular}{|l|c|c|}
\hline & SVM & DCNN \\
\hline Accuracy & 98.92 & 99.25 \\
\hline Precision & 98.67 & 98.97 \\
\hline Recall & 99.33 & 99.63 \\
\hline F-Score & 98.99 & 99.29 \\
\hline
\end{tabular}




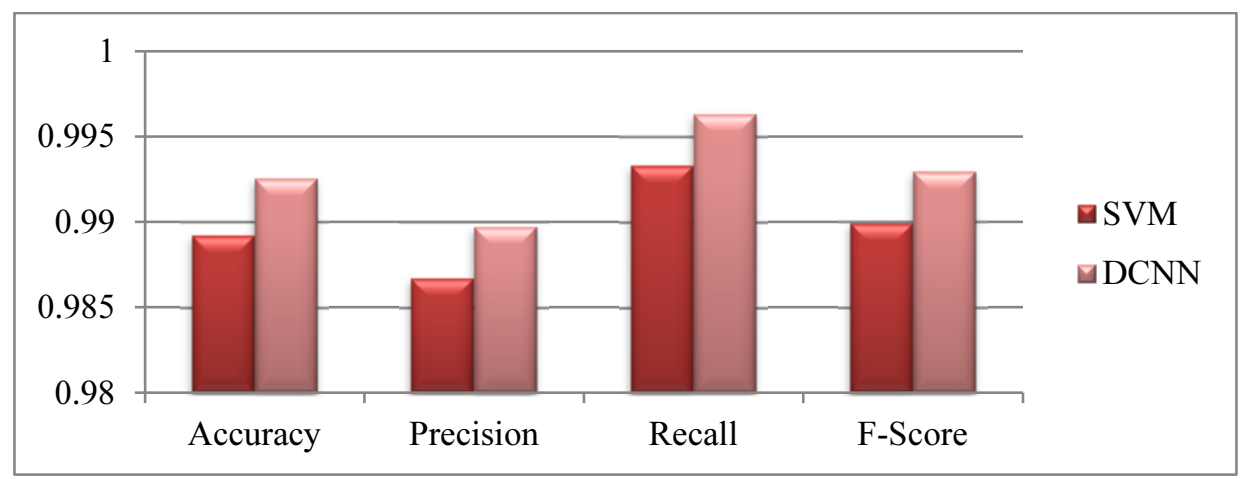

Figure 2. Performance evaluation using Naïve Bayes and SVM

According to above Figure 2, we demonstrate Deep CNN provides better detection accuracy over the Naïve Bayes machine learning algorithm. The CNN has used multi convolutional layers and collaboration with activation function.

\section{Conclusions}

The primary purpose was to use underwater image processing techniques. For the pipeline network, the same error was found in each file. This research is to be presented. Corrosion on the inside and out has a multitude of different causes, but the structural integrity is required in order to ensure that all of them are considered. Anomalous pipeline wear deposits on this pipeline construction pipeline can be examined to identify pipeline flaw carriers that originate from the excavation of galvanized steel. The mechanism of corrosion is the formation of a galvanic couple between microbe-produced iron sulphides and steel. Using the available information, we can apply this Machine Learning methodology to determine the corrosion. For subpip corrosion analysis, a new approach for pip drawing will be implemented using images. Before performing a self-collected reconstruction and improvement, an initial self-check was performed to rule out self-diagnose issues. It can be inferred from the publicly accessible underwater imagery dataset, and it appears that the two are very promising. The experiment was done alongside the exploration of the color drift of the traditional picture measurements. The research will provide data for risk assessment models used for maintenance repair and functions of the pipeline system. The bald question asks the rest of the question some possible perspectives on the safe life of the tube can be applied or developed Studying rust-related cracking and pitting events.

\section{References}

[1] Khan A, Ali SS, Anwer A, Adil SH, Mériaudeau F. Subsea Pipeline Corrosion Estimation by Restoring and Enhancing Degraded Underwater Images. IEEE Access. 2018 Jul 13;6.40585-601.

[2] Rankin A, Ivanov T, Brennan S. Evaluating the performance of unmanned ground vehicle water detection. InProceedings of the 10th Performance Metrics for Intelligent Systems Workshop 2010 Sep 28 (pp. 305-311).

[3] L.Developments in sonar technologies and their applications. In2013 IEEE International Underwater Technology Symposium (UT) 2013 Mar 5 (pp. 1-8). IEEE.

[4] Szyrowski T, Sharma SK, Sutton R, Kennedy GA. Developments in subsea power and telecommunication cables detection. Part 1-Visual and hydroacoustic tracking. Underwater Technology. 2013 Jul 1;31(3). 
[5] Bonnin-Pascual F, Ortiz A. On the use of robots and vision technologies for the inspection of vessels. A survey on recent advances. Ocean Engineering. 2019 Oct 15;190.106420.

[6] Motamedi M, Faramarzi F, Duran O. New concept for corrosion inspection of urban pipeline networks by digital image processing. InIECON 2012-38th Annual Conference on IEEE Industrial Electronics Society 2012 Oct 25 (pp. 1551-1556). IEEE.

[7] Potharaju, S. P., \& Sreedevi, M. (2018). A novel cluster of quarter feature selection based on symmetrical uncertainty. Gazi University Journal of Science, 31(2), 456-470.

[8] Gao, Y.; Liu, Y.; Ma, Y.; Cheng, X.; Yang, J. Application of the differentiation process into the correlation-based leak detection in urban pipeline networks. Mech. Syst. Signal Process. 2018, $112,251-264$.

[9] Yin, S.; Weng, Y.; Song, Z.; Cheng, B.; Gu, H.; Wang, H.; Yao, J. Mass transfer characteristics of pipeline leak-before-break in a nuclear power station. Appl. Therm. Eng. 2018, 142, 194-202.

[10] Scott, S.L.; Barrufet, M.A. Worldwide Assessment of Industry Leak Detection Capabilities for Single \& Multiphase Pipelines. Offshore Technology Research Center College Station. 2003. Available online. http.//citeseerx.ist.psu.edu/viewdoc/download?doi=10.1.1.118.6455\&rep=rep1\& type $=$ pdf (accessed on 17 December 2018) 